Texas A\&M University-San Antonio

Digital Commons @ Texas A\&M University-San Antonio

$5-2021$

\title{
The Effects of Low Volume Versus High Volume Sled-Push Training on Muscular Adaptation
}

Jeffrey R. Bernard

Yi-Hung Liao

Christian 0. Madrigal

Joshua D. Levesque

Matthew B. Fraze

See next page for additional authors

Follow this and additional works at: https://digitalcommons.tamusa.edu/kin_faculty

Part of the Kinesiology Commons 
Authors

Jeffrey R. Bernard, Yi-Hung Liao, Christian O. Madrigal, Joshua D. Levesque, Matthew B. Fraze, Isaac Del Torro, and Sukho Lee 


\title{
The Effects of Low Volume Versus High Volume Sled-Push Training on Muscular Adaptation
}

\author{
Jeffrey R. Bernard $\mathrm{PhD}^{1}$, Yi-Hung Liao $\mathrm{PhD}^{2}$, Christian O. Madrigal BA' ${ }^{1}$, Joshua D. Levesque MS ${ }^{1}$, Matthew B. Fraze MS ${ }^{1}$, \\ Isaac Del Toro $\mathrm{BA}^{1}$, Sukho Lee $\mathrm{PhD}^{3}$ \\ ${ }^{1}$ Department of Kinesiology, California State University, Stanislaus, Turlock, CA, USA; ${ }^{2}$ Department of Exercise and Health Science, National Taipei University of Nursing \\ and Health Sciences, Taipei, Taiwan; ${ }^{3}$ Department of Counseling, Health, and Kinesiology, Texas A\&M University- San Antonio, San Antonio, TX, USA
}

PURPOSE: This study aimed to compare the effects of low-volume and high-volume sled-push resistance training on muscle strength, power, and body composition.

METHODS: Twenty-four college students were recruited and matched based on baseline one-repetition maximum (1-RM) into one of the three groups: 1) low volume (LV) resistance training, 2) high volume (HV) resistance training, or 3) control (CON) (n=8 per group). The LV training consisted of five single repetitions of pushing a weighted sled for $9.1 \mathrm{~m}$. The HV training consisted of three sets of five repetitions of pushing a weighted sled for $9.1 \mathrm{~m}$. Training consisted of three weekly workouts performed on nonconsecutive days for 6 weeks. This study utilized a pre-test and post-test design consisting of 1-RM, Wingate power test, standing long jump, vertical jump, and body composition.

RESULTS: After 6 weeks of training, there was a similar but significant increase in 1-RM in both training groups (pre-test: $\mathrm{LV}=226.8 \pm 14.8 \mathrm{~kg}$ vs. $\mathrm{HV}=217.7 \pm 19.5 \mathrm{~kg}$; post-test: $\mathrm{LV}=298.5 \pm 15 \mathrm{~kg}$ vs. $\mathrm{HV}=286.9 \pm 16 \mathrm{~kg}, p<.001)$. However, no improvements were observed in the Wingate power test, standing long jump, vertical jump, or body composition in both training and CON groups $(p>.05)$.

CONCLUSIONS: The results suggested that low-volume resistance training was as effective as a high-volume protocol for improving muscle strength. However, the present study was unable to determine the effects on muscle power and body composition.

Key words: Resistance training, Sled-push training, Muscle strength testing, Muscle power testing, Body composition.

\section{INTRODUCTION}

Various resistance training protocols have been utilized by coaches, personal trainers, and health professionals in pursuit of improving muscular strength, power, and body composition. To produce positive muscle gains the limits of skeletal muscle must be challenged, or overloaded. For example, according to Henneman's size principle, higher thresholds must be reached by larger loads for greater motor unit recruitment and increased strength development [1]. Thus, high resistance stress must be placed on skeletal muscle fibers in order for an overload stimulus to be present, leading to muscular adaptations.
Until recently lifting greater loads were viewed as the optimal means to induce the required overload stimulus. However, as sports training has evolved to include more functional movements, alternative ways to achieve overload have been employed. Therefore, the combination of manipulating intensity, frequency, and/or volume has become more common. One way to balance frequency and volume is to set intensity as a percent of one's maximal repetition (1-RM). Thus, at a low percentage of 1-RM one can perform a high frequency and volume, while as one gets closer to their 1-RM, both frequency and volume greatly decrease [2]. However, it is not entirely clear which combination of intensity, frequency, and volume are best, how these can be manipulated to

Corresponding author: Sukho Lee Tel +1-210-784-2537 Fax +1-210-784-2523 E-mail sleelatamusa.edu

Received 17 Feb 2021 Revised 17 May 2021 Accepted 26 May 2021

@)This is an Open Access article distributed under the terms of the Creative Commons Attribution Non-Commercial License (https://creativecommons.org/licenses/by-nc/4.0/) which permits unrestricted non-commercial use, distribution, and reproduction in any medium, provided the original work is properly cited. 
achieve the same gains, or if the same principles can be applied to all modes of exercise.

As muscle mass increases in response to a resistance training program, there is often times an associated improvement in body composition. The increase in lean body mass will offset fat mass, resulting in a decrease in body fat percentage. A recent short duration study employing a strongman training style that incorporated sled-push work reported a positive change in body composition [3]. Thus, resistance training, at least when an overload stimulus is provided, can increase muscle mass relative to changes in fat mass. However, it is still not clear whether low and high volume sled-push resistance training produce different impacts on muscle strength, power, and body composition or not. We selected sled-push training because this mode of exercise has received little attention in the literature, yet is a commonly applied training method used by both novice and experienced resistance trained individuals.

Therefore, the purpose of this study was to evaluate the effectiveness of low and high volume sled-push resistance training protocols on muscular strength, power, and body composition. Based on previous work [4], we hypothesized that the low volume resistance training protocol would induce similar improvements as the high-volume resistance training protocol.

\section{METHODS}

\section{Study Design}

A pre- and post-training study design was applied to determine the effects of sled-push training. A 1-repitition max (1-RM) consisting of a $9.1 \mathrm{~m}$ (equivalent to 10 yards) sled-push was used to pair participants into either low volume (LV) resistance training, high volume (HV) resistance training, or control (CON) treatment groups. After initial groups were matched each subject underwent several other pre- and post-tests to assess body composition, and anaerobic/muscular power. Lower body muscular power was assessed using both the standing long jump and vertical jump tests via a Just Jump system (Probotics Inc., Huntsville, AL). Anaerobic power was also assessed using a standardized Wingate anaerobic power test on a Veltron Dynafit Pro cycle ergometer (RacerMate Inc., Seattle WA). Body composition was evaluated via bioelectrical impedance analysis using a handheld fat loss monitor (Omron, Hoffman Estates, IL).

\section{Subjects}

Twenty-four recreationally trained college age students were recruited to participate in this study. All study procedures were approved by the University Institutional Review Board (UIRB) at California State University, Stanislaus. Subjects were informed of the benefits and risks of the investigation prior to signing an institutionally approved informed consent document to participate in the study. All subjects were deemed low risk in accordance to the American College of Sports Medicine (ACSM) Health History Questionnaire. Subjects were pair matched based on their baseline 1-RM into 1 of 3 treatment groups: 1) LV, 2) HV, or 3) CON ( $n=8$ per group). Subjects were instructed to not exercise outside of the study training sessions and to maintain a similar diet throughout the duration of the study.

\section{Methodology}

Each training group exercised for 6-weeks with 3 non-consecutive training sessions each week. Both training groups were instructed to push a weighted sled non-stop for $9.1 \mathrm{~m}$. The LV training protocol followed an ascending cluster set consisting of 5 total repetitions: 90, 93, 95, 100 , and $105 \%$ of their 1-RM. Thirty seconds of rest was allowed between each repetition. The final weight completed was recorded as a new maximum weight and used as a maximal weight to calculate training weight for the subsequent training session. If the subject didn't complete their final repetition, they returned to the same training protocol the next session until they could complete the protocol. The HV training protocol consisted of 3 sets of 5 repetitions: $85 \%, 87 \%$, and $90 \%$ of their 1-RM. Thirty seconds of rest was allowed between repetitions and a total of 2-minute between each set. If the subject completed the final set at 90\% that became their new 1-RM for the next training session.

\section{Statistical Analysis}

All data sets were analyzed using SPSS software (SPSS Inc., Chicago, IL., USA). One-way analysis of variance (ANOVA) was used to compare means. If a significant interaction was identified, means were compared using a Fisher's least significant difference post hoc test. A level of $p<.05$ was deemed statistically significant. All values are expressed as means \pm slandered error of mean (SEM).

\section{RESULTS}

The pre- and post-test height, weight, and body composition were 


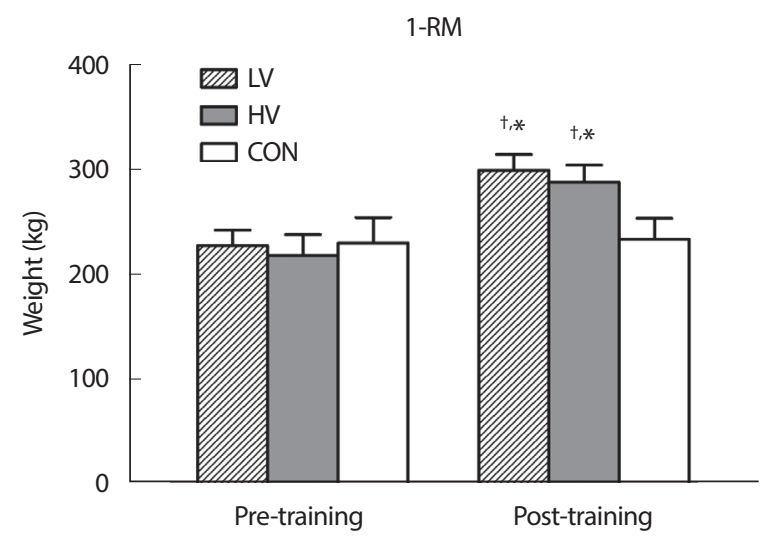

Fig. 1. Sled-push 1-RM values before and after 6-weeks of training. Low Volume ( $\mathrm{LV}, \mathrm{n}=8)$, High Volume ( $\mathrm{HV} \mathrm{n}=8)$, and Control ( $\mathrm{CON}, \mathrm{n}=8)$. Values are means \pm SEM. ${ }^{*} p<.001$, from pre-test value. ${ }^{\dagger} p<.001$, from post-test $\mathrm{CON}$.

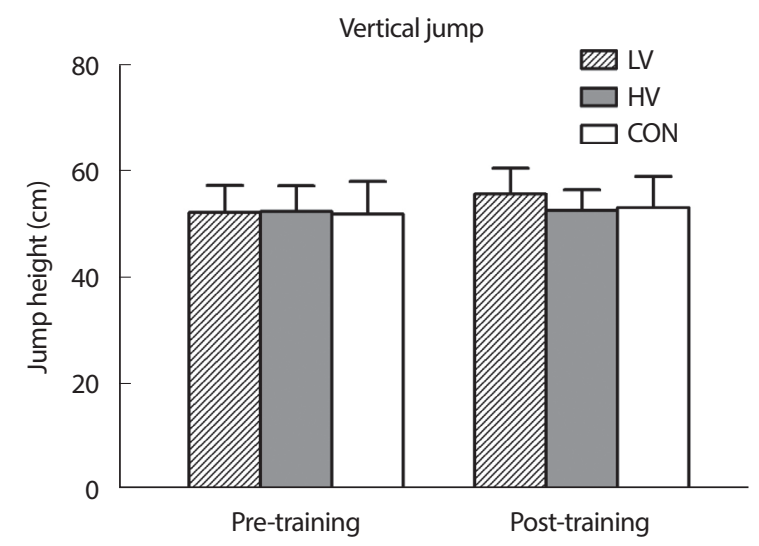

Fig. 2. Vertical jump values before and after 6-weeks of training. Low Volume ( $(\mathrm{VV}, \mathrm{n}=8)$, High Volume ( $\mathrm{HV} \mathrm{n}=8)$, and Control (CON, $\mathrm{n}=8)$. Values are means \pm SEM.

Table 1. Subject characteristics

\begin{tabular}{lcccc}
\hline & Age $(\mathrm{yr})$ & Height $(\mathrm{cm})$ & Weight $(\mathrm{kg})$ & Body Fat (\%) \\
\hline LV & $21.8 \pm 1.0$ & $26 \pm 0.4$ & $74.8 \pm 4.5$ & $20.9 \pm 3.5$ \\
HV & $21.9 \pm 0.5$ & $25.7 \pm 0.4$ & $71.1 \pm 5.1$ & $21.6 \pm 3.6$ \\
CON & $22.3 \pm 1.1$ & $26.1 \pm 0.6$ & $73.8 \pm 4.6$ & $22.3 \pm 1.1$ \\
\hline
\end{tabular}

Pre-test age, height, weight, and \% body fat for the LV (Low Volume), HV (High Volume), and Control (CON) groups.

similar between all treatment groups and were unchanged at the end of the study (Table 1, only pre-test data shown). Following 6-weeks of sledpush training, both the LV and HV groups significantly increased their maximum strength (1-RM) (pre-test; $\mathrm{LV}=226.8 \pm 14.8 \mathrm{~kg}$ vs. HV $217.7 \pm$ $19.5 \mathrm{~kg}$; post-test; $\mathrm{LV}=298.5 \pm 15 \mathrm{~kg}$ vs. $\mathrm{HV}=286.9 \pm 16 \mathrm{~kg}, p<.001)$, while there was no change observed among the CON group. Interestingly, although both training groups increased their 1-RM, there was no significant difference in the increased strength between the LV and HV train-

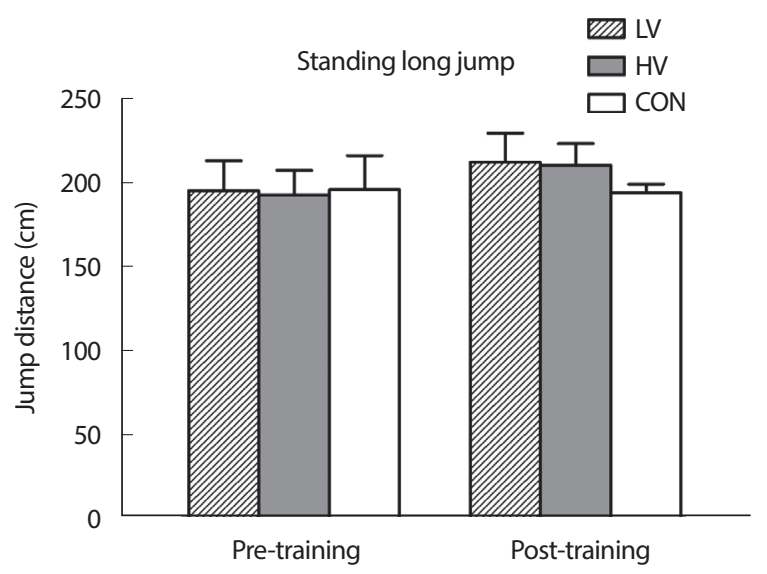

Fig. 3. Long jump values before and after 6-weeks of training. Low Volume $(\mathrm{LV}, \mathrm{n}=8)$, High Volume (HV $\mathrm{n}=8)$, and Control $(\mathrm{CON}, \mathrm{n}=8)$. Values are means \pm SEM.

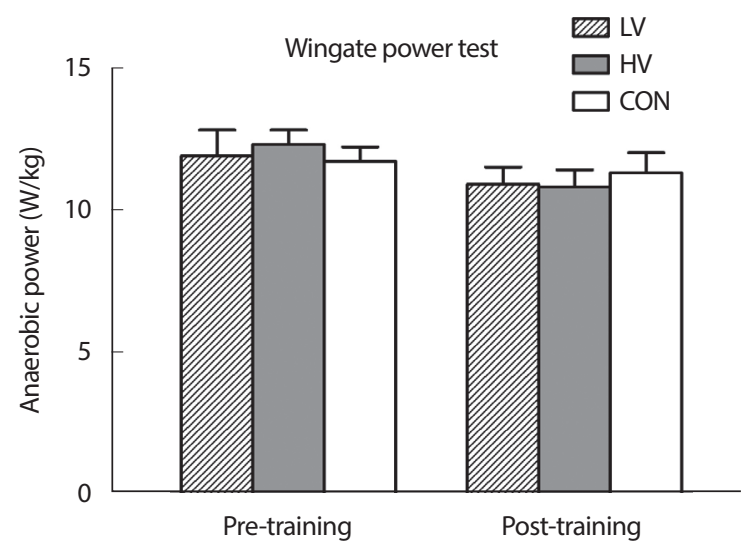

Fig. 4. Anaerobic power as determine by the Wingate power test before and after 6 -weeks of training. Low Volume (LV, $n=8)$, High Volume (HV $\mathrm{n}=8)$, and Control $(\mathrm{CON}, \mathrm{n}=8)$. Values are means \pm SEM.

ing groups (Fig. 1).

There was no significant difference or change in the vertical jump between the three groups when pre- and post-tests were compared (Fig. 2). Although there was a trend indicating improved pre- versus post-test vertical and standing long jump performance among the LV and HV training groups, the improvement was not statistically significant (Fig. 3). Lastly, neither the LV nor HV training groups demonstrated improved anaerobic performance, as determined by the Wingate power test, between pre- and post-test measurements (Fig. 4). The CON group did not show any improvement for the muscular power tests.

\section{DISCUSSION}

The purpose of this study was to evaluate the effects of both low and high volume sled-push resistance training protocols on muscle strength, 
power, and body composition. Even though the training programs did not induce significant difference in muscular adaptations between training groups, both the LV and HV training groups increased their 1-RM in the $9.1 \mathrm{~m}$ sled-push. Thus, the present investigation agrees with our previous report [4] that a LV training protocol is as effective as a standard HV training protocol in improving muscle force. This suggests that similar increases in strength can be achieved in a short period of time utilizing a low volume high intensity training program. This may be more time efficient as opposed to the high volume training protocol utilized in this study. The increase in force production resulting from a strength training program is related to various mechanisms including morphological adaptations, changes in maximal motor unit discharge rate, and changes at the whole-muscle and single-fiber levels, among others. The results of the present study suggest that during the first three weeks of training, a low-volume and high-volume of strength training may induce a similar stimulus under the mechanism related to strength increases.

Unfortunately, neither of the training groups experienced significant changes in body weight nor body composition. However, Winwood et al. [3] reported that the combination of sled-pushing with strongman style training did, improved body composition. This may be due to the strongman-training program utilizing several exercises to incorporate a full body-training regimen. Collectively, this would suggest that sledpush training alone may not be suitable to improve body composition. Rather a range of full body-training, whether it be traditional or nontraditional exercises, is more appropriate for enhancing body composition.

It has been suggested that the muscle hypertrophy may not be evident until at least 6 or more weeks of training [5]. Our study was short in duration and highly specific. Therefore, we do not want to rule out entirely that muscle hypertrophy did not occur in our study. Rather, it is plausible that short term training programs, such as ours, may induce some hypertrophy. In support of this, it has been reported that lower body and upper body hypertrophy do not occur at the same rate. A study by Abe et al. [6] found that increases in muscle mass can be detected as early as 6-weeks for the upper body whereas hypertrophy may be delayed in the lower body [6]. Nevertheless, more advanced means of assessment may be required. For example, both Dual-energy X-ray absorptiometry DEXA and magnetic imaging resonance (MRI) have been used to assess muscle mass and could possibly detect early changes in muscle cross sectional area $[7,8]$.
Being that sled-push training did not affect body composition in the present investigation, this would suggest that the strength increases observed in this study were most likely related to neuromuscular improvements rather than muscular hypertrophy. Despite the vigor of both training protocols, it is plausible that the short training duration (i.e. 6-weeks) of the program was not long enough to observe hypertrophy in this subject population. This is not entirely surprising based on what has been reported previously by others and what is currently known about muscle physiology $[9,10]$. Thus, it is likely that utilizing the same training protocol for a longer duration would result in further strength gains that could be attributed to muscle hypertrophy.

Recently, a 6-week study compared the effects of weightlifting, kettlebell, and traditional resistance training modalities on body composition and vertical jump performance [11]. Similar to what we found, Otto et al. [11] reported no positive changes in body composition following 6-weeks of resistance training. The authors suggested that short-term training sessions, such as ours, can only elicit neuromuscular adaptations. Thus, a 6-week training protocol is not long enough to experience significant muscle growth. However, Otto et al. [11] did report that both weightlifting and kettlebell training increased vertical jump performance, with weightlifting being superior over the kettlebells. The authors equate this performance increase to the similarity of kettlebell exercises and the specific weightlifting exercises to that of the vertical jump [11], both of which differ from sled-pushing. In contrast to sled-push training, the specific weightlifting and kettlebell exercises used in their studies, incorporated the same lower body musculature that is used during the vertical jump. Furthermore, both modalities also incorporate high velocity movements similar to that of the vertical jump. Unlike the horizontal displacement of pushing a sled, their specific weightlifting and kettlebell training incorporated high velocity vertical displacement of a load. Therefore, it is likely that our sled-push training may not have been specific enough to increase vertical jump performance due to its slower velocity horizontal displacement.

Although we observed significant increases in muscle force (as determined be 1-RM) as a result of sled-push training, we did not find an improvement in muscle power. Changes in anaerobic power may not be seen in short duration training interventions due to anaerobic capacity being dependent on chronic physiological adaptations. In contrast, a recent study reported a positive change in anaerobic power following a short 6-week training protocol utilizing both traditional power training and high intensity circuit style power training [12]. Both training meth- 
ods improved power output on a standardized Wingate test. This suggests that anaerobic power can increase following a short training protocol where much of the adaptations are neurological. Yet again, our sledpush training, which involved moving heavy loads at a self-selected pace, may have not been specific enough to trigger these changes in power. Thus, instructing one to push the sled as fast as possible may be sufficient to induce increases in muscle power. Therefore, more research is necessary to determine if sled-push training with high velocity can be as effective as traditional power training for improving anaerobic power.

Despite the interesting findings of this study, some limitations should be noted. First of all, the relatively small sample size for each group may have influenced the data. Thus, it's possible that the small sample size masked some of the physiological adaptations that would become evident had we involved more participants. In addition, the sled-push training protocol used in the present study may have impacted the results. The lack of previous research utilizing sled-push training made it difficult to follow a proven training program. Lastly, the participants only trained for 6-week. It's feasible that additional weeks of training would elicit improvements associated with changes in body composition and differences in other variables between the LV and HV training groups.

\section{Practical Applications}

This research shows that sled-push training can be considered a viable option to increase athletic performance and/or supplement a traditional resistance-training program. Sled-pushing is a functional, locomotive movement that involves lower leg extension, which can be used as a strength training exercise (as shown here) or even as an explosive movement or sprint. For example, sled pushing can be utilized for sports that involve driving an opponent such as American football, rugby, or Mixed Martial Arts. Thus, non-traditional strength training methods, such as sled-push training, can elicit significant strength gains to improve performance and offer variability in training to enhance engagement and motivation.

\section{CONCLUSIONS}

In conclusion, this study demonstrated that a low volume sled-push training program is as effective as a high volume program to significantly increase muscle force production. However, improving force under these conditions may be more related to neuromuscular adaptations rather than improvements in body composition and/or muscle hyper- trophy. Although sled-push training increases muscle force, it appears that this type of training may not be applicable for improvements in muscle power. Thus, further research is warranted to study the benefits of sled-push training and how it can be used to improve athletic performance.

\section{CONFLICT OF INTEREST}

The authors of this paper do not declare any conflict of interest.

\section{AUTHOR CONTRIBUTIONS}

Conceptualization: JRB MBF SL YHL; Data curation: COM JDL JRB IDT MBF; Formal analysis: JRB YHL SL; Funding acquisition; Methodology: JRB MBF SL YHL; Project administration: JRB SL; Visualization; Writing-original draft: JDL JRB YHL; Writing-review \& editing: COM JDL JRB IDT MBF SL YHL.

\section{ORCID}

$\begin{array}{ll}\text { Yi-Hung Liao } & \text { https://orcid.org/0000-0003-1958-6112 } \\ \text { Sukho Lee } & \text { https://orcid.org/0000-0002-8506-2897 }\end{array}$

\section{REFERENCES}

1. Houtman CJ, Stegeman DF, Van Dijk JP, Zwarts MJ. Changes in muscle fiber conduction velocity indicate recruitment of distinct motor unit populations. J Appl Physiol. 2003;95(3):1045-54. DOI: 10.1152/ japplphysiol.00665.2002

2. Richens B, Cleather DJ. The relationship between the number of repetitions performed at given intensities is different in endurance and strength trained athletes. Biol Sport. 2014;31(2):157-61. DOI: 10.5604/ 20831862.1099047

3. Winwood PW, Cronin JB, Posthumus LR, Finlayson SJ, Gill ND. Strongman vs. traditional resistance training effects on muscular function and performance. J Strength Cond Res. 2015;29(2):429-39. DOI: 10.1519/ JSC.0000000000000629

4. Yoon A, Hong JY, Choi SM, Hahn DW, Kim KJ, et al. Comparison of multiple sets and progressive single set of resistance training on muscle mass and strength in rat. Exerc Sci. 2015;24(3):267-74. DOI: 10.15857/ ksep.2015.24.3.267 
5. Bompa T, Buzzichelli CA. Periodization training for sports. 3rd ed., Champaign: Human Kinetics 2015.

6. Abe T, DeHovos DV, Pollock ML, Garzarella L. Time course for strength and muscle thickness changes following upper and lower body resistance training in men and women. Eur J Appl Physiol. 2000; 81(3):174-80. DOI: $10.1007 / \mathrm{s} 004210050027$

7. DeFreitas, JM, Beck TW, Stock MS, Dillion MA, Kasishke PR. An examination of the time course of training-induced skeletal muscle hypertrophy. Eur J Appl Physiol. 2011;111(11):2785-90. DOI: 10.1007/ s00421-011-1905-4

8. Maden-Wilkinson TM, Degens H, Jones DA, McPhee JS. Comparison of MRI and DXA to measure muscle size and age-related atrophy in thigh muscles. J Musculoskelet Neuronal Interact. 2013;13(3):320-8.

9. Folland JP, Williams AG. The adaptations to strength training: mor- phological and neurological contributions to increased strength. Sports Med. 2007;37(2):145-68. DOI: 10.2165/00007256-200737020-00004

10. Gabriel DA, Kamen G, Frost G. Neural adaptations to resistive exercise: mechanisms and recommendations for training practices. Sports Med. 2006;36(2):133-49. DOI: 10.2165/00007256-200636020-00004

11. Otto WH, Coburn JW, Brown LE, Spiering BA. Effects of weightlifting vs. kettlebell training on vertical jump, strength, and body composition. J Strength Cond Res. 2012;26(5):1199-202. DOI: 10.1519/JSC. 0b013e31824f233e

12. Romero-Arenas S, Ruiz R, Vera A, Colomer-Poveda D, Grau AG, et al. Neuromuscular and cardiovascular adaptations in response to high intensity interval power training. J Strength Cond Res. 2018;32(1):1308. DOI: 10.1519/JSC.0000000000001778 